\title{
The Effects of Heterogeneous Spatial Networks in Multi-agent Parrondo's Games
}

\author{
Junyong Shu, Keren Rui, Ye Ye*, Lu Wang and Nenggang Xie \\ School of Mechanical Engineering, Anhui University of Technology, Ma'anshan, Anhui Province, 243002, China \\ ${ }^{*}$ Corresponding author
}

\begin{abstract}
In the multi-agent Parrondo's games, if game $\mathrm{A}$ and game $B$ both take certain network structures, then the heterogeneity of the network will produce impacts. By using the analytical approach based on the discrete Markov chain, we analyzed a one-dimensional case. Then, we deduced the transition probability matrix of game $A$ under one dimensional line (homogeneous with game B) and a fully-connected network (heterogeneous with game $B$ ). Moreover, we gave the mathematical expectation of the randomized game $A+B$. The theoretical results showed that for the one-dimensional case the heterogeneity between game $A$ and game $B$ enlarges the parameter space of the strong paradox. Besides, we performed calculation simulations on two-dimensional networks. We used the following four networks for game A: a two-dimensional lattice (homogeneous with game B), random network, a scale-free network and a fully-connected network (the latter three networks are heterogeneous with game $B$ ). The simulation results showed that the gain and the strong paradox space both decrease with the increment of the degree of the heterogeneous, which shows that homogeneity between game $A$ and game $B$ is beneficial for twodimensional networks.
\end{abstract}

Keywords-parrondo's paradox, parrondo's games, complex networks, heterogeneity

\section{INTRODUCTION}

Parrondo's paradox is an apparent paradox in game theory, and it is named after its creator Parrondo, a Spanish physicist [1]. The seminal papers on Parrondo's paradox were published by Abbott and Harmer [2-3] in 1999. Moreover, Parrondo's paradox has been developed into many different versions [4]. Generally, Parrondo's games involve two games, A and B. Game B has some branches, some of which are favorable (that is the probability of winning is large) and others are unfavorable (that is the probability of losing is large). The design of the branches requires some forms of dependence. At present, according to the available Parrondo's games, the dependent mechanisms mainly include three types, which are based on the individual capital, on the individual history of experience and on neighbors' environment. According to the size of the participants, there mainly includes individual and group versions. Toral [8] proposed a space-dependent "cooperative Parrondo's paradox" version. A remarkable difference is that there are $i(i=2, \ldots, N)$ players instead of only one player involved in the game. However, here the multiagent version was not carried out. Game A remained unchanged as was defined in the original Parrondo's games. Game B, which was based on the neighboring environment. Wang [11] presented a version of Parondox's paradox played in a population of agents where game A was the original game based on the flip of a suitably biased coin and game B was a 2 $\times 2$ game played between a pair of agents selected randomly. In this version, game B showed the characteristics of the multiagent game, that is, individuals had interactive roles between themselves. As the previous versions have focused on how to modify game B, Toral [12] proposed a modification of game A. There were $N$ players involved in this version as well and there was interaction between individuals for game A. Game B remained unchanged as defined in the original Parrondo's games or in the history-dependent version. In the version proposed by Xie [13], game A was the same as that in Toral [12] and game $B$ was played based on the neighboring environment. Here, game A and game B produced the coupling effects spatially.

For game $\mathrm{A}$ in the multi-agent versions, individuals have a interactive relationship, which belongs to a zero-sum game from the view of the population level. There are two key points in the interactive relationship: 1) The interactive modes. They can be divided into competition and cooperation. In the version proposed by Toral [12], individuals used the cooperative mode. Wang [14] presented four kinds of interaction, which were competition, cooperation, harmony and PCRC, respectively. 2) The network carriers. The population has a certain spatial distribution or a spatial structure which will be abstracted into a certain topology network in the theoretical analysis. Individuals interact through networks. In the version proposed by Toral [12], the network carriers were the fully-connected network and a one-dimensional line. Xie [13] also used a onedimensional line. Wang [14] and Ye [15-16] adopted a fullyconnected network, random network and a BA scale-free network as their spatial carriers. Moreover, they obtained that different network structures and the heterogeneity have different effects on the multi-agent Parrondo's games.

In the above studies of Toral [12], Wang [14] and Ye [1516], game A belonged to the multi-agent version and they use the capital-dependent and history-dependent mechanisms for game $\mathrm{B}$. If game $\mathrm{B}$ was based on the neighboring environment mechanism, then game A and game B had the coupling effects. There are many network structures for game A while there are only one dimensional line and a two-dimensional lattice for game B due to the structure limitation. Xie [13] analyzed the multi-agent Parrondo's game under the homogeneous networks. However, there are no relevant studies at present when game A and game B have different network carriers. For such a case, the paper demonstrates the effects of the heterogeneity of 
networks through the theoretical and computer simulation analysis.

\section{The TheORETICAL ANALysis BASED ON A ONE- DIMENSIONAL LINE}

\section{A. Model}

The multi-agent Parrondo's model based on one dimensional line is shown in Figure I [13]. Here, game B uses one dimensional line and game $\mathrm{A}$ uses the following two networks: 1) one dimensional line (the same as game B); 2) a fully connected network (heterogeneous with game B). Consider a population made up of $N$ individuals. On each round of game, one player ' $i$ ' is randomly chosen from $N$ persons to randomly play game A with probability $\gamma$ or B with probability $1-\gamma)$, then the result is either winning(expressed by 1) or losing(expressed by 0 ). When game $\mathrm{A}$ is played, individual $j$ is chosen from $i$ 's spatial neighbors randomly (for the one-dimensional line, Player $i$ 's neighbors are $i-1$ and $i+1$; for the fully-connected network, all players are $i$ 's neighbors except ' $i$ ' itself). Then the interaction between $i$ and $j$ is assumed to be a competitive way. The probabilities that $i$ and $j$ win are 0.5 , respectively. $j$ pays $\$ 1$ for $i$ when $i$ wins; Otherwise, $i$ pays $\$ 1$ for $j$. When game B is played, the winning and losing probabilities of individual $i$ are dissimilar under different conditions of neighboring environment. Player $i$ 's neighbors are players $i-1$ and $i+1$ who have four different kinds of winning and losing states. Therefore, game B is composed of 4 branches. The corresponding winning probabilities of the individual $i$ are $p_{\mathrm{h}}(h=0,1,2,3)$. Winning game B earns $\$ 1$ and losing surrenders $\$ 1$.

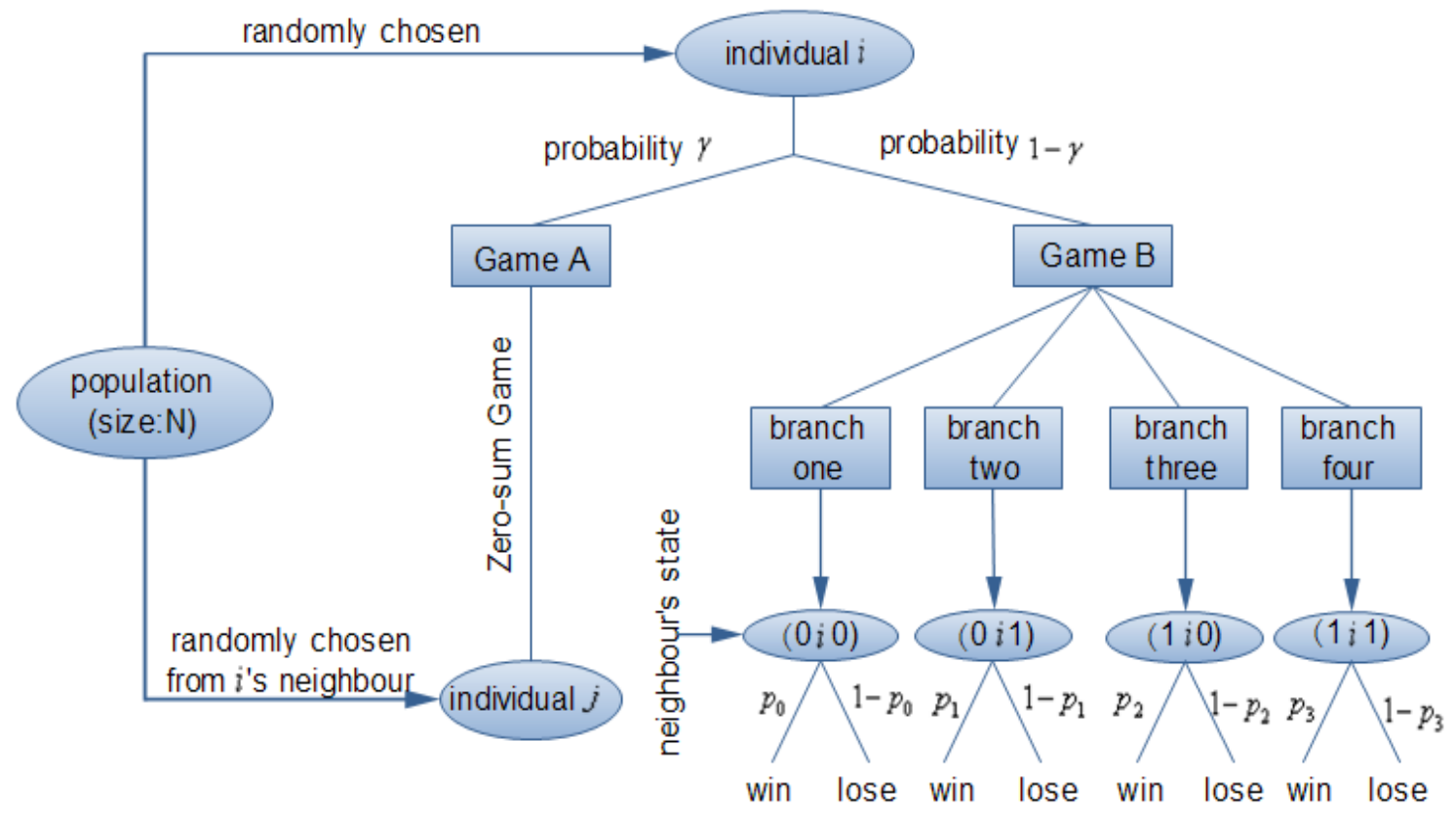

FIGURE I. THE MULTI-AGENT PARRONDO'S MODEL BASED ON A ONE-DIMENSIONAL LINE

\section{B. The Analysis of Game A}

Four individuals $(N=4)$ are played in game A for example. There are 16 states$\mathrm{E}=\{(0000),(0001),(0010),(0011), \ldots,(1110),(1111)\}$, which are $0,1,2,3,4, \ldots \ldots, 14,15$, in decimal notations correspondingly. Therefore, the state set can also be denoted by $E=\{0,1,2, \ldots, 14,15\}$. The transition probability matrix is $[P]^{(A)}$.

$$
[P]^{(A)}=\left[p_{a b}\right]_{a, b \in E}=\left[\begin{array}{cccc}
p_{00} & p_{01} & \cdots & p_{0-15} \\
p_{10} & p_{11} & \cdots & p_{1-15} \\
\vdots & \vdots & \vdots & \vdots \\
p_{15-0} & p_{15-1} & \cdots & p_{15-15}
\end{array}\right]
$$

1) $[\mathrm{P}]^{(\mathrm{A})}$ in the one-dimensional network: When game $\mathrm{A}$ is played on the one-dimensional network, any individual i can only compete with its two neighbors, $i-1$ and $i+1$. Taking $p_{15}$ as an example, we explain the calculation method on the elements of this matrix. Because game $\mathrm{A}$ is a game between two neighboring individuals, thus there are only 4 categories, that is, individuals 1 and 2, individuals 2 and 3, individuals 3 and 4 , and individuals 4 and 1 . From state $1(0001)$ to state 5 (0101), two cases exist. One is that individual 1 and individual 2 compete and individual 2 wins (The winning probability is $1 / 2$ ), and the probability of occurrence is $\frac{1}{1} \times \frac{1}{2}=\frac{1}{8}$. The other is that individual 2 and individual 3 cotnpete, individual 2 wins (The winning probability is $1 / 2$ ), and the probability of occurrence is $\frac{1}{1} \times \frac{1}{2}=\frac{1}{8}$. Therefore, $p_{15}=\frac{1}{8}+\frac{1}{8}=\frac{1}{1}$. Then other matrix eftements $\overline{\& a n}_{\text {an }}$ be calculated in the satme way. Finally we obtain the transition probability matrix of game A [13] 


$$
[P]^{(A)}=\left[\begin{array}{cccccccccccccccc}
0 & 1 / 4 & 1 / 4 & 0 & 1 / 4 & 0 & 0 & 0 & 1 / 4 & 0 & 0 & 0 & 0 & 0 & 0 & 0 \\
0 & 1 / 4 & 1 / 8 & 1 / 8 & 0 & 1 / 4 & 0 & 0 & 1 / 8 & 1 / 8 & 0 & 0 & 0 & 0 & 0 & 0 \\
0 & 1 / 8 & 1 / 4 & 1 / 8 & 1 / 8 & 0 & 1 / 8 & 0 & 0 & 0 & 1 / 4 & 0 & 0 & 0 & 0 & 0 \\
0 & 1 / 8 & 1 / 8 & 1 / 4 & 0 & 1 / 8 & 0 & 1 / 8 & 0 & 0 & 1 / 8 & 1 / 8 & 0 & 0 & 0 & 0 \\
0 & 0 & 1 / 8 & 0 & 1 / 4 & 1 / 4 & 1 / 8 & 0 & 1 / 8 & 0 & 0 & 0 & 1 / 8 & 0 & 0 & 0 \\
0 & 0 & 0 & 1 / 8 & 0 & 1 / 2 & 1 / 8 & 0 & 0 & 1 / 8 & 0 & 0 & 1 / 8 & 0 & 0 & 0 \\
0 & 0 & 1 / 8 & 0 & 1 / 8 & 1 / 8 & 1 / 4 & 1 / 8 & 0 & 0 & 1 / 8 & 0 & 0 & 0 & 1 / 8 & 0 \\
0 & 0 & 0 & 1 / 8 & 0 & 1 / 4 & 1 / 8 & 1 / 4 & 0 & 0 & 0 & 1 / 8 & 0 & 0 & 1 / 8 & 0 \\
0 & 1 / 8 & 0 & 0 & 1 / 8 & 0 & 0 & 0 & 1 / 4 & 1 / 8 & 1 / 4 & 0 & 1 / 8 & 0 & 0 & 0 \\
0 & 1 / 8 & 0 & 0 & 0 & 1 / 8 & 0 & 0 & 1 / 8 & 1 / 4 & 1 / 8 & 1 / 8 & 0 & 1 / 8 & 0 & 0 \\
0 & 0 & 0 & 1 / 8 & 0 & 0 & 1 / 8 & 0 & 0 & 1 / 8 & 1 / 2 & 0 & 1 / 8 & 0 & 0 & 0 \\
0 & 0 & 0 & 1 / 8 & 0 & 0 & 0 & 1 / 8 & 0 & 1 / 8 & 1 / 4 & 1 / 4 & 0 & 1 / 8 & 0 & 0 \\
0 & 0 & 0 & 0 & 1 / 8 & 1 / 8 & 0 & 0 & 1 / 8 & 0 & 1 / 8 & 0 & 1 / 4 & 1 / 8 & 1 / 8 & 0 \\
0 & 0 & 0 & 0 & 0 & 1 / 4 & 0 & 0 & 0 & 1 / 8 & 0 & 1 / 8 & 1 / 8 & 1 / 4 & 1 / 8 & 0 \\
0 & 0 & 0 & 0 & 0 & 0 & 1 / 8 & 1 / 8 & 0 & 0 & 1 / 4 & 0 & 1 / 8 & 1 / 8 & 1 / 4 & 0 \\
0 & 0 & 0 & 0 & 0 & 0 & 0 & 1 / 4 & 0 & 0 & 0 & 1 / 4 & 0 & 1 / 4 & 1 / 4 & 0
\end{array}\right]
$$

The corresponding stationary distribution probability of game $\mathrm{A}$ is

$\{\pi\}^{(A)}=\{0,1 / 24,1 / 24,1 / 12,1 / 24,1 / 6,1 / 12,1 / 24,1 / 24,1 / 12,1 / 6$, $1 / 24,1 / 12,1 / 24,1 / 24,0\}$.

2) $[P]^{(A)}$ in the fully-connected network: When game A uses the fully-connected network, any individual i can compete with all its neighbors. Taking $\mathrm{p}_{15}$ as an example, we explain the calculation method on the elements of this matrix. As game $\mathrm{A}$ is played between any two individuals, thus there are $C_{4}^{2}=6$ categories. From state $1(0001)$ to state $5(0101)$, two cases exist. One is that individual 1 and individual 2 compete and individual 2 wins (The winning probability is $1 / 2)$, and the probability of occurrence is $\frac{1}{6} \times \frac{1}{2}=\frac{1}{\text {. The }}$ other is that individual 2 and individual 3 compete, inAividual 2 wins (The winning probability is $1 / 2$ ), and the probability of occurrence is $\frac{1}{6} \times \frac{1}{2}=\frac{1}{12}$. Therefore, $p_{15}=\frac{1}{12}+\frac{1}{12}=\frac{1}{1}$. Then other matrix Elements 1 ean be calculated it2 the ${ }^{2}$ sanfe way. Finally we can obtain the transition probability matrix of game A.

$$
[P]^{(A)}=\left[\begin{array}{cccccccccccccccc}
0 & 1 / 4 & 1 / 4 & 0 & 1 / 4 & 0 & 0 & 0 & 1 / 4 & 0 & 0 & 0 & 0 & 0 & 0 & 0 \\
0 & 1 / 4 & 1 / 12 & 1 / 6 & 1 / 12 & 1 / 6 & 0 & 0 & 1 / 12 & 1 / 6 & 0 & 0 & 0 & 0 & 0 & 0 \\
0 & 1 / 12 & 1 / 4 & 1 / 6 & 1 / 12 & 0 & 1 / 6 & 0 & 1 / 12 & 0 & 1 / 6 & 0 & 0 & 0 & 0 & 0 \\
0 & 1 / 12 & 1 / 12 & 1 / 3 & 0 & 1 / 12 & 1 / 12 & 1 / 12 & 0 & 1 / 12 & 1 / 12 & 1 / 12 & 0 & 0 & 0 & 0 \\
0 & 1 / 12 & 1 / 12 & 0 & 1 / 4 & 1 / 6 & 1 / 6 & 0 & 1 / 12 & 0 & 0 & 0 & 1 / 6 & 0 & 0 & 0 \\
0 & 1 / 12 & 0 & 1 / 12 & 1 / 12 & 1 / 3 & 1 / 12 & 1 / 12 & 0 & 1 / 12 & 0 & 0 & 1 / 12 & 1 / 12 & 0 & 0 \\
0 & 0 & 1 / 12 & 1 / 12 & 1 / 12 & 1 / 12 & 1 / 3 & 1 / 12 & 0 & 0 & 1 / 12 & 0 & 1 / 12 & 0 & 1 / 12 & 0 \\
0 & 0 & 0 & 1 / 6 & 0 & 1 / 6 & 1 / 6 & 1 / 4 & 0 & 0 & 0 & 1 / 12 & 0 & 1 / 12 & 1 / 12 & 0 \\
0 & 1 / 12 & 1 / 12 & 0 & 1 / 12 & 0 & 0 & 0 & 1 / 4 & 1 / 6 & 1 / 6 & 0 & 1 / 6 & 0 & 0 & 0 \\
0 & 1 / 12 & 0 & 1 / 12 & 0 & 1 / 12 & 0 & 0 & 1 / 12 & 1 / 3 & 1 / 12 & 1 / 12 & 1 / 12 & 1 / 12 & 0 & 0 \\
0 & 0 & 1 / 12 & 1 / 12 & 0 & 0 & 1 / 12 & 0 & 1 / 12 & 1 / 12 & 1 / 3 & 1 / 12 & 1 / 12 & 0 & 1 / 12 & 0 \\
0 & 0 & 0 & 1 / 6 & 0 & 0 & 0 & 1 / 12 & 0 & 1 / 6 & 1 / 6 & 1 / 4 & 0 & 1 / 12 & 1 / 12 & 0 \\
0 & 0 & 0 & 0 & 1 / 12 & 1 / 12 & 1 / 12 & 0 & 1 / 12 & 1 / 12 & 1 / 12 & 0 & 1 / 3 & 1 / 12 & 1 / 12 & 0 \\
0 & 0 & 0 & 0 & 0 & 1 / 6 & 0 & 1 / 12 & 0 & 1 / 6 & 0 & 1 / 12 & 1 / 6 & 1 / 4 & 1 / 12 & 0 \\
0 & 0 & 0 & 0 & 0 & 0 & 1 / 6 & 1 / 12 & 0 & 0 & 1 / 6 & 1 / 12 & 1 / 6 & 1 / 12 & 1 / 4 & 0 \\
0 & 0 & 0 & 0 & 0 & 0 & 0 & 1 / 4 & 0 & 0 & 0 & 1 / 4 & 0 & 1 / 4 & 1 / 4 & 0
\end{array}\right]
$$

The corresponding stationary distribution probability of game $\mathrm{A}$ is
$\{\pi\}^{(A)}=\{0,1 / 20,1 / 20,1 / 10,1 / 20,1 / 10,1 / 10,1 / 20,1 / 20,1 / 10,1 / 1$ $0,1 / 20,1 / 10,1 / 20,1 / 20,0\}$. 
No matter what network carrier is used, game A is a zerosum game, so the probability of winning or losing is 0.5 regardless of the state. So the mean probability of the overall winning in game $\mathrm{A}$ is

$$
\{\lambda\}_{\text {win }}^{(A)}=\left\{\begin{array}{lllll}
0.5 & 0.5 & \cdots & 0.5 & 0.5
\end{array}\right\}^{T}
$$

\section{The Analysis of Game B}

According to the method proposed by Mihailovic [9], we can obtain the transition probability matrix of game $\mathrm{B}$ for a case with $N=4$.

The transition probability matrix of game $\mathrm{B}[13]$ is

$$
[P]^{(B)}=\frac{1}{4}\left(\begin{array}{cccccccccccccccc}
4 p_{0}^{1} & p_{0} & p_{0} & 0 & p_{0} & 0 & 0 & 0 & p_{0} & 0 & 0 & 0 & 0 & 0 & 0 & 0 \\
p_{0}^{1} & Q_{1} & 0 & p_{1} & 0 & p_{0} & 0 & 0 & 0 & p_{2} & 0 & 0 & 0 & 0 & 0 & 0 \\
p_{0}^{1} & 0 & Q_{1} & p_{2} & 0 & 0 & p_{1} & 0 & 0 & 0 & p_{0} & 0 & 0 & 0 & 0 & 0 \\
0 & p_{1}^{1} & p_{2}^{1} & 2 & 0 & 0 & 0 & p_{1} & 0 & 0 & 0 & p_{2} & 0 & 0 & 0 & 0 \\
p_{0}^{1} & 0 & 0 & 0 & Q_{1} & p_{0} & p_{2} & 0 & 0 & 0 & 0 & 0 & p_{1} & 0 & 0 & 0 \\
0 & p_{0}^{1} & 0 & 0 & p_{0}^{1} & Q_{2} & 0 & p_{3} & 0 & 0 & 0 & 0 & 0 & p_{3} & 0 & 0 \\
0 & 0 & p_{1}^{1} & 0 & p_{2}^{1} & 0 & 2 & p_{2} & 0 & 0 & 0 & 0 & 0 & 0 & p_{1} & 0 \\
0 & 0 & 0 & p_{1}^{1} & 0 & p_{3}^{1} & p_{2}^{1} & Q_{3} & 0 & 0 & 0 & 0 & 0 & 0 & 0 & p_{3} \\
p_{0}^{1} & 0 & 0 & 0 & 0 & 0 & 0 & 0 & Q_{1} & p_{1} & p_{0} & 0 & p_{2} & 0 & 0 & 0 \\
0 & p_{2}^{1} & 0 & 0 & 0 & 0 & 0 & 0 & p_{1}^{1} & 2 & 0 & p_{1} & 0 & p_{2} & 0 & 0 \\
0 & 0 & p_{0}^{1} & 0 & 0 & 0 & 0 & 0 & p_{0}^{1} & 0 & Q_{2} & p_{3} & 0 & 0 & p_{3} & 0 \\
0 & 0 & 0 & p_{2}^{1} & 0 & 0 & 0 & 0 & 0 & p_{1}^{1} & p_{3}^{1} & Q_{3} & 0 & 0 & 0 & p_{3} \\
0 & 0 & 0 & 0 & p_{1}^{1} & 0 & 0 & 0 & p_{2}^{1} & 0 & 0 & 0 & 2 & p_{1} & p_{2} & 0 \\
0 & 0 & 0 & 0 & 0 & p_{3}^{1} & 0 & 0 & 0 & p_{2}^{1} & 0 & 0 & p_{1}^{1} & Q_{3} & 0 & p_{3} \\
0 & 0 & 0 & 0 & 0 & 0 & p_{1}^{1} & 0 & 0 & 0 & p_{3}^{1} & 0 & p_{2}^{1} & 0 & Q_{3} & p_{3} \\
0 & 0 & 0 & 0 & 0 & 0 & 0 & p_{3}^{1} & 0 & 0 & 0 & p_{3}^{1} & 0 & p_{3}^{1} & p_{3}^{1} & 4 p_{3}
\end{array}\right)
$$

where: $p_{h}^{1}=1-p_{h}, h=0,1,2,3 ; Q_{1}=3-p_{1}-p_{2}$;

$$
Q_{2}=2-2 p_{3}+2 p_{0} ; Q_{3}=1+p_{1}+p_{2} \text {. }
$$

The mathematic expectation of game $B$ is

$$
E^{(B)}=\{\pi\}^{(B)}\left(\{\lambda\}_{\text {win }}^{(B)}-\{\lambda\}_{\text {lose }}^{(B)}\right)
$$

where: $\{\pi\}^{(B)}$ is the stationary distribution probability of game B. It can be obtained by solving $\{\boldsymbol{\pi}\}^{(B)}=\{\boldsymbol{\pi}\}^{(B)}[P]^{(B)}$, where $[P]^{(B)}$ is the transition probability matrix of game $\mathrm{B}$. $\{\lambda\}_{\text {win }}^{(B)}$ and $\{\lambda\}_{\text {lose }}^{(B)}$ are the average probabilities of the overall winning and losing in game $\mathrm{B}$, respectively. $\{\lambda\}_{\text {lose }}^{(\mathrm{B})}=I-\{\lambda\}_{\text {win }}^{(\mathrm{B})} . I=\left\{\begin{array}{llll}1 & 1 & \cdots & 1\end{array}\right\}$.

$\{\lambda\}_{\text {win }}^{(B)}=\left\{\begin{array}{llll}\lambda_{0 w}^{(B)} & \lambda_{1 w}^{(B)} & \cdots & \lambda_{15 w}^{(B)}\end{array}\right\}^{T}$ are the average probabilities of the overall winning from state 1 to state 15 in game B. Xie gave the calculation method to solve $\lambda_{i w}^{(B)}$ in Ref.

\begin{tabular}{|c|c|c|c|c|c|c|c|c|}
\hline state & 0000 & 0001 & 0010 & 0011 & 0100 & 0101 & 0110 & 0111 \\
\hline$\lambda_{i w}^{(B)}$ & $\mathrm{p}_{0}$ & $\begin{array}{c}\left(\mathrm{p}_{2}+\mathrm{p}_{0}+\mathrm{p}_{1}\right. \\
\left.+\mathrm{p}_{0}\right) / 4\end{array}$ & $\begin{array}{c}\left(\mathrm{p}_{0}+\mathrm{p}_{1}+\mathrm{p}_{0}\right. \\
\left.+\mathrm{p}_{2}\right) / 4\end{array}$ & $\begin{array}{c}\left(\mathrm{p}_{2}+\mathrm{p}_{1}+\mathrm{p}_{1}\right. \\
\left.+\mathrm{p}_{2}\right) / 4\end{array}$ & $\begin{array}{c}\left(\mathrm{p}_{1}+\mathrm{p}_{0}+\mathrm{p}_{2}\right. \\
\left.+\mathrm{p}_{0}\right) / 4\end{array}$ & $\begin{array}{c}\left(\mathrm{p}_{3}+\mathrm{p}_{0}+\mathrm{p}_{3}\right. \\
\left.+\mathrm{p}_{0}\right) / 4\end{array}$ & $\begin{array}{c}\left(\mathrm{p}_{1}+\mathrm{p}_{1}+\mathrm{p}_{2}\right. \\
\left.+\mathrm{p}_{2}\right) / 4\end{array}$ & $\begin{array}{c}\left(\mathrm{p}_{3}+\mathrm{p}_{1}+\mathrm{p}_{3}\right. \\
\left.+\mathrm{p}_{2}\right) / 4\end{array}$ \\
\hline state & 1000 & 1001 & 1010 & 1011 & 1100 & 1101 & 1110 & 1111 \\
\hline$\lambda_{i w}^{(B)}$ & $\begin{array}{c}\left(\mathrm{p}_{0}+\mathrm{p}_{2}+\mathrm{p}_{0}\right. \\
\left.+\mathrm{p}_{1}\right) / 4\end{array}$ & $\begin{array}{c}\left(\mathrm{p}_{2}+\mathrm{p}_{2}+\mathrm{p}_{1}\right. \\
\left.+\mathrm{p}_{1}\right) / 4\end{array}$ & $\begin{array}{c}\left(\mathrm{p}_{0}+\mathrm{p}_{3}+\mathrm{p}_{0}\right. \\
\left.+\mathrm{p}_{3}\right) / 4\end{array}$ & $\begin{array}{c}\left(\mathrm{p}_{2}+\mathrm{p}_{3}+\mathrm{p}_{1}\right. \\
\left.+\mathrm{p}_{3}\right) / 4\end{array}$ & $\begin{array}{c}\left(\mathrm{p}_{1}+\mathrm{p}_{2}+\mathrm{p}_{2}\right. \\
\left.+\mathrm{p}_{1}\right) / 4\end{array}$ & $\begin{array}{c}\left(\mathrm{p}_{3}+\mathrm{p}_{2}+\mathrm{p}_{3}\right. \\
\left.+\mathrm{p}_{1}\right) / 4\end{array}$ & $\begin{array}{c}\left(\mathrm{p}_{1}+\mathrm{p}_{3}+\mathrm{p}_{2}\right. \\
\left.+\mathrm{p}_{3}\right) / 4\end{array}$ & $\mathrm{p}_{3}$ \\
\hline
\end{tabular}
[13]. Table I shows the average probability $\lambda_{i w}^{(B)}$ of the overall winning from state 1 to state 15 in game $B$.

TABLE I. THE AVERAGE PROBABILITY OF THE OVERALL WINNING OF GAME B

D. The Analysis of the Randomized Game $A+B$

The transition probability matrix of the randomized game $\mathrm{A}+\mathrm{B}$ is

$$
[P]^{\operatorname{rank}(A+B)}=\gamma \cdot[P]^{(A)}+(1-\gamma) \cdot[P]^{(B)}
$$

where: $\gamma$ is the probability of playing game A. 
According to (8), we deduce the stationary distribution probability $\{\pi\}^{\operatorname{rank}(A+B)}$ of the randomized game $\mathrm{A}+\mathrm{B}$.

$$
\{\boldsymbol{\pi}\}^{\operatorname{rank}(A+B)}=\{\boldsymbol{\pi}\}^{\operatorname{rank}(A+B)}[P]^{\operatorname{rank}(A+B)}
$$

The mathematical expectation of the game $\mathrm{A}+\mathrm{B}$ is $E^{\operatorname{rank}(A+B)}$

$$
E^{\operatorname{rank}(A+B)}=\{\pi\}^{\operatorname{rank}(A+B)}\left(\{\lambda\}_{\operatorname{win}}^{\operatorname{rank}(A+B)}-\{\lambda\}_{\text {lose }}^{\operatorname{rank}(A+B)}\right)
$$

where: $\{\lambda\}_{\text {win }}^{\operatorname{rank}(A+B)}$ and $\{\lambda\}_{\text {lose }}^{\operatorname{rank}(A+B)}$ are the average probabilities of the overall winning and losing in game $\mathrm{A}+\mathrm{B}$. Moreover, $\quad\{\lambda\}_{\text {lose }}^{\operatorname{rank}(A+B)}=I-\{\lambda\}_{\text {win }}^{\operatorname{rank}(A+B)}$ $I=\left\{\begin{array}{llll}1 & 1 & \cdots & 1\end{array}\right\}$.

$$
\{\lambda\}_{\text {win }}^{\operatorname{rank}(A+B)}=\gamma \cdot\{\lambda\}_{\text {win }}^{(A)}+(1-\gamma) \cdot\{\lambda\}_{\text {win }}^{(B)}
$$

where: $\{\lambda\}_{\text {win }}^{\{A)}$ and $\{\lambda\}_{\text {win }}^{\{B)}$ are the mean probabilities of the overall winning in game $\mathrm{A}$ and game $\mathrm{B}$.

The established conditions of the weak paradoxical form is

$$
\begin{gathered}
E^{\operatorname{rank}(A+B)} \geq E^{(B)} \\
E^{(B)} \leq 0
\end{gathered}
$$

The established conditions of the strong paradoxical form is

$$
\begin{gathered}
E^{\operatorname{rank}(A+B)} \geq 0 \\
E^{(B)} \leq 0
\end{gathered}
$$

\section{E. The Analysis of Results}

We take $N=4$ and get the mathematical expectation of game B according to $[P]^{(B)}$ in (5) and $\{\lambda\}_{\text {win }}^{(B)}=\left\{\begin{array}{llll}\lambda_{0 w}^{(B)} & \lambda_{1 w}^{(B)} & \cdots & \lambda_{15 w}^{(B)}\end{array}\right\}^{T}$ in Table 1. Then, based on $[P]^{(A)}$ in (2) and (3) and $[P]^{(B)}$ in (5), we obtain $\{\pi\}^{\operatorname{rank}(A+B)}$ by using (7) and (8). Moreover, by substituting $\{\lambda\}_{\text {win }}^{\{A)}$ in (4) and $\{\lambda\}_{\text {win }}^{\{B)}$ in (5) into (10), we get $\{\lambda\}_{\text {win }}^{\operatorname{rank}(A+B)}$. Finally, we substitute $\{\pi\}^{\operatorname{rank}(A+B)}$ and $\{\lambda\}_{\text {win }}^{\operatorname{rank}(A+B)}$ into (9) and obtain the mathematical expectation of playing the randomized game $\mathrm{A}+\mathrm{B}$. The calculation results of Figure II show that when game
A uses one dimensional line (homogeneous with game B), the parameter space where the strong paradox occurs is smaller than that by using the fully-connected network (heterogeneous with game B). Therefore, for the one dimensional line, the heterogeneity between game A and game B expands the parameter space where strong paradox occurs.

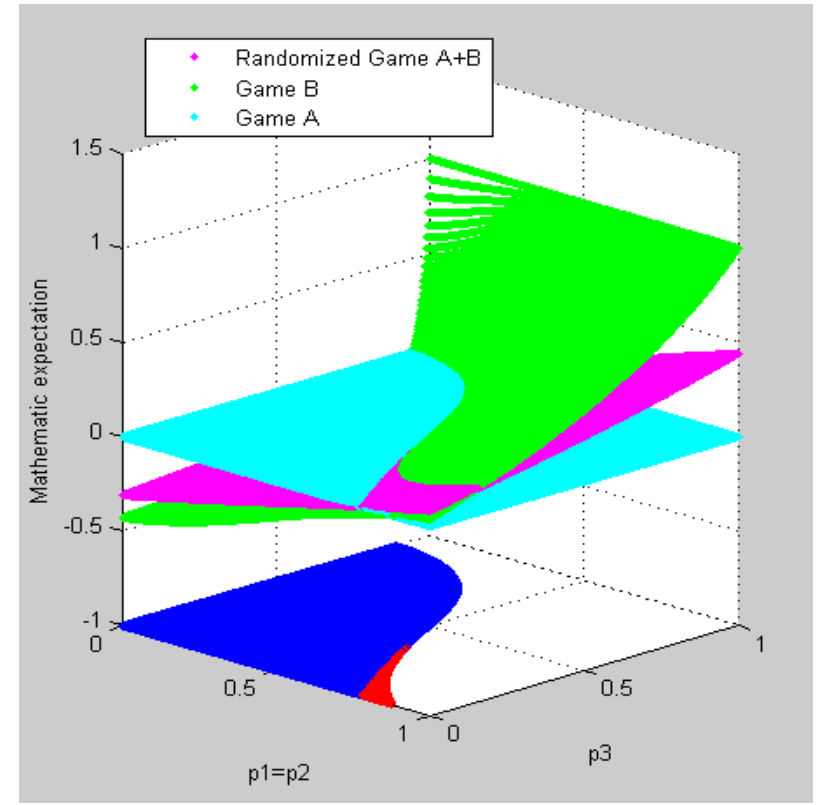

a. a one-dimensional line

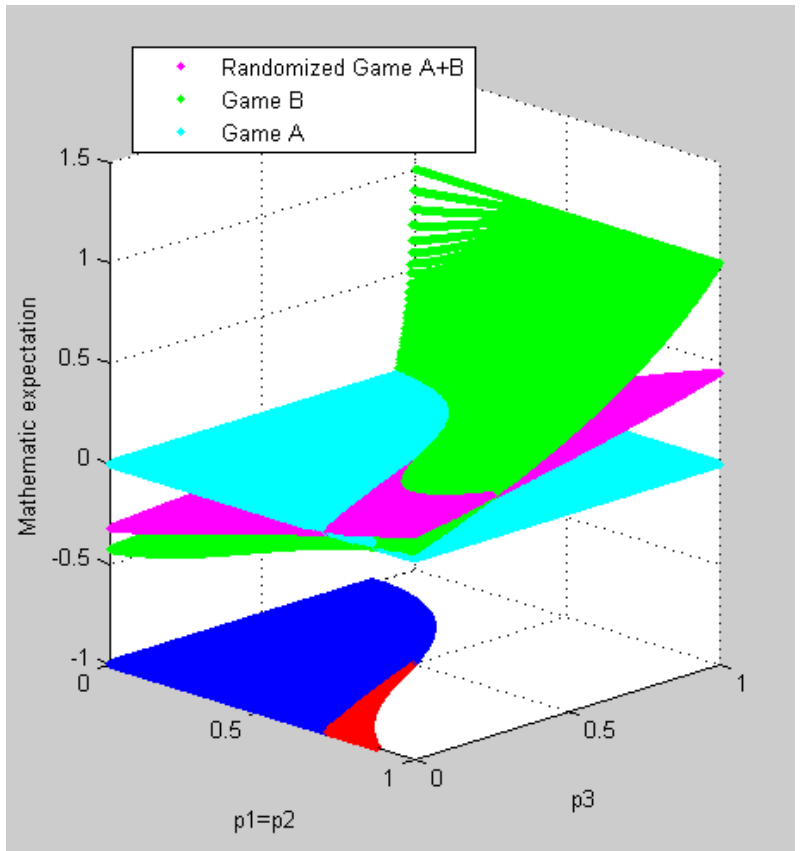

b. a fully-connected network

FIGURE II. THE THEORETICAL RESULTS OF PARRONDO'S GAMES BASED ON A ONE DIMENSIONAL LINE (A: GAME A USES A ONE-DIMENSIONAL LINE. B: GAME A USES A FULLYCONNECTED NETWORK. THE POPULATION SIZE IS N $=4$. $\Gamma=$ $0.5, \mathrm{P} 0=0.5$. THE BLUE AREA DENOTES THE PARAMETERS AREA OF WEAK PARRONDO'S PARADOX WHILE THE RED STANDS FOR THE STRONG ONE). 


\section{THE SIMULATION ANALYSIS BASED ON A TWO- DIMENSIONAL LATTICE}

\section{A. Model}

The multi-agent model based on a two-dimensional lattice is shown in Figure III[16], where game B uses two dimensional lattice ( such a square lattice uses periodic boundary conditions, that is, the left and right edges are connected; the up and below edges are connected.). Game A uses the following networks: 1) a two-dimensional lattice (homogeneous with game B); 2) random network (heterogeneous with game B); a scale-free network (heterogeneous with game B); 4) a fully connected network. On each round of game, one player ' $i$ ' is randomly chosen from $N$ players to randomly play game A (probability $\gamma$ ) or B (probability $1-\gamma$ ). When game A is played, individual $j$ is chosen from $i$ 's spatial neighbors randomly. Then the interaction between $i$ and $j$ is assumed in a competitive way. When game $\mathrm{B}$ is played, the winning or losing probability of individual $i$ is dissimilar under different conditions of neighboring environment, which has five different kinds of winning and losing conditions. Therefore, game B is composed of 5 branches. The corresponding winning probabilities of the individual $i$ are $p_{h}(h=0,1,2,3,4)$.

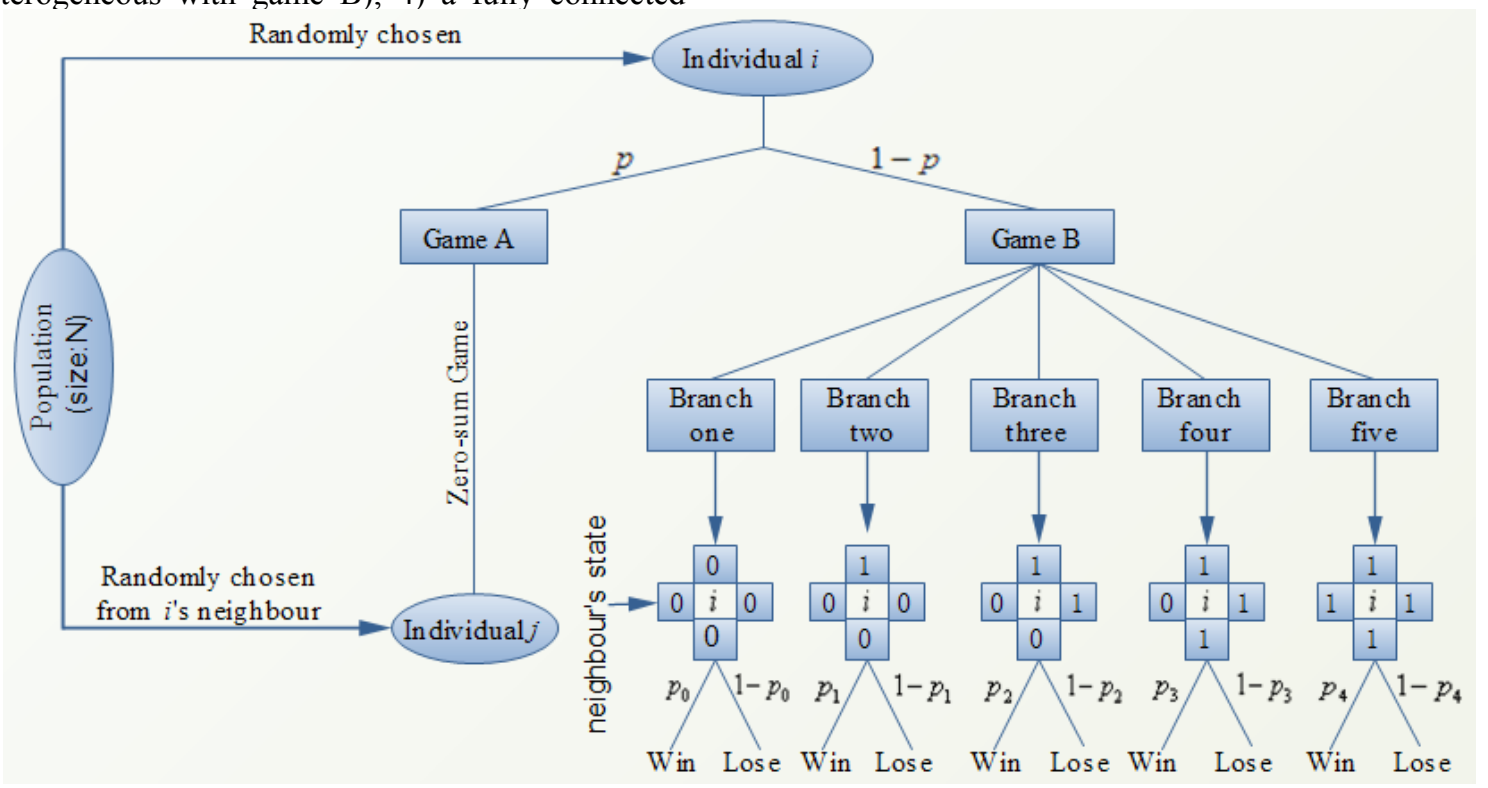

FIGURE III. THE MULTI-AGENT PARRONDO'S MODEL BASED ON A TWO DIMENSIONAL LATTICE

\section{B. Construction of Random Network}

We use the rewiring mechanism [17] to generate random network. Based on the original square lattice, first a randomly chosen $E F$ link is broken up and the site $E$ is rewired to the randomly chosen site $D(D$ cannot be the neighbor of $E)$. This process is repeated $K$ times. Compare random network with the original square lattice and the degree of the heterogeneity is determined by the rewiring times $K$.

\section{Construction of a BA Scale-Free Network}

To demonstrate the progressively changing from random network to scale-free network, we use an adjustable-parameter network of degree distribution and the corresponding construction method. The steps are in [18]: a) growth. The initial network consists of $N$ nodes, where $m_{0}$ nodes are fully connected among themselves. Thus, the set $J_{2}$ is constructed; the unconnected set $J_{1}$ is composed by $\left(\left(N-m_{0}\right)\right.$ isolated nodes. At each time step, choose one new node from $J_{1}$. In order to produce $m$ edges, we connect this new node to some other nodes. b) preferential connectivity. $m$ edges of the new node are connected to the nodes from the remaining $(N-1)$ nodes with probability $\lambda$; then connect the nodes of the set $J_{2}$ by following a linear preferential attachment strategy with the probability $1-\lambda$. When the connectivity is completed, we remove the new node from $J_{1}$ and add it to $J_{2}$ c) After $N-m_{0}$ steps, the network model is generated by a series of parameter $\lambda \in[0,1]$, where $\lambda=0$ corresponds to a BA scale-free network and $\lambda=1$ corresponds to random network.

\section{The Calculation Results and the Analysis}

For the two dimensional lattice, we also perform the theoretical analysis. Without generality, we analyze the case of $N=9$ at least (the $3 \times 3$ lattice network). With the increase of $N$, the transition probability matrix is added with $2^{N}$. Then, the analysis of the stationary distribution probability and the mathematical expectation becomes difficult. Therefore, for the two dimensional lattice, we mainly use computer simulations to analyze.

For computer simulations, we define the multi-agent average fitness $d(t)$ as

$$
d(t)=\frac{W(t)}{T}
$$


where: $W(t)=\sum_{i=1}^{N}\left[C_{i}(t)-C_{0}\right]$ was the multi-agent total profit. $C_{i}(t)$ was the capital of individual $i$ at time $t, C_{0}$ was the initial capital and $T$ was the total time of the game.

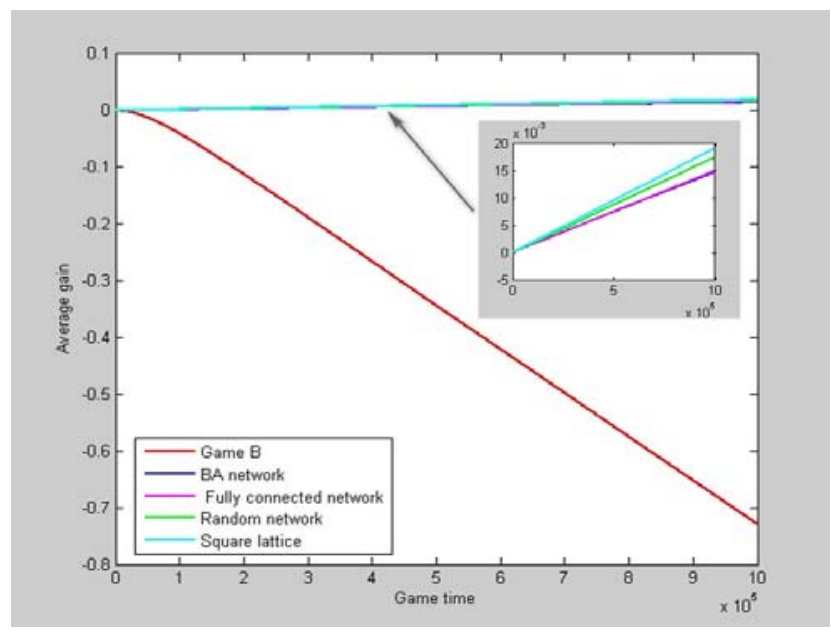

FIGURE IV. SIMULATION RESULTS (THE POPULATION SIZE N IS 1600 AND PLAYING TIME T IS 100 000. THE INITIAL WINNING (1) AND LOSING (0) STATES ARE SET RANDOMLY. THE PROBABILITY OF PLAYING GAME A IS $\mathrm{P}=0.5$ AND THE PARAMETERS OF GAME B ARE P0 $=0.01, \mathrm{P} 1=0.15, \mathrm{P} 2=\mathrm{P} 3=0.7$ AND P4 $=0.6$. WE USE DIFFERENT RANDOM NUMBERS TO PLAY THE GAME FOR 100 TIMES REPETITIVELY AND THEN MAKE A FIGURE FROM THE AVERAGE VALUE OF THEIR RESULTS. THE REWIRING TIMES K IS 1600 IN RANDOM NETWORK.)

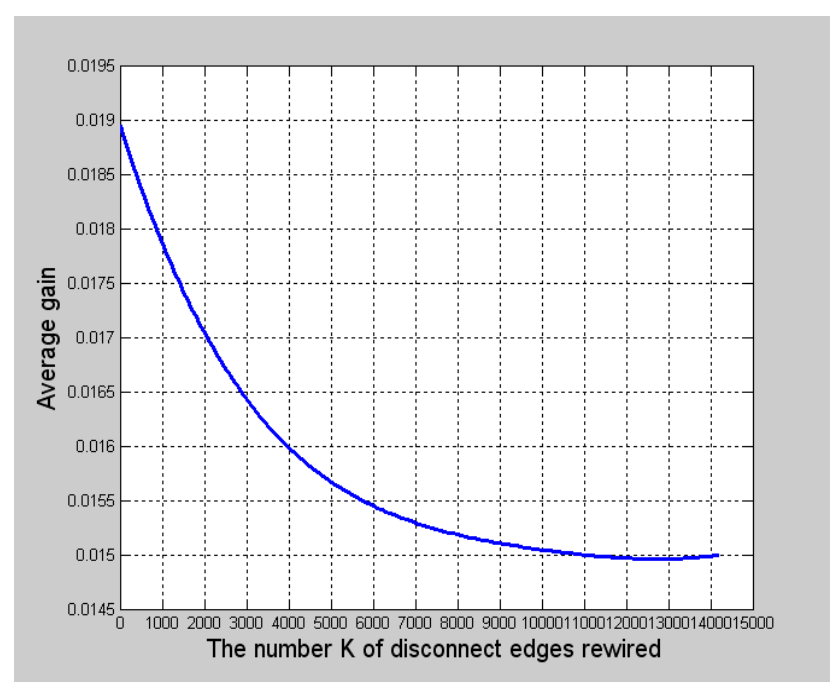

FIGURE V. THE EFFECT THAT THE HETEROGENIETY OF THE NETWORKS (THAT IS THE REWIRING TIMES K) HAS ON THE POPULATION GAIN (THE NETWORK CARRIER OF GAME A CHANGES PROGRESSIVELY FROM REGULAR NETWORK TO RANDOM NETWORK WITH THE INCREMENT OF K. OTHER PARAMETER ARE THE SAME AS IN FIGURE 4.)

Figure IV shows the simulation results of the average gain of the population, including playing game B individually (game
$\mathrm{B}$ uses a square lattice) and the randomized game $\mathrm{A}+\mathrm{B}$ (where game A uses a square lattice, random network, a BA scale-free network and a fully-connected network, respectively). According to Figure IV, we notice that no matter what network game $\mathrm{A}$ uses, the gain of the randomized game $\mathrm{A}+\mathrm{B}$ is positive, where Parrondo's paradox occurs. Especially, the gain based on the square lattice is the largest. Therefore, for the square lattice, when game A and game B have the same network, they are conducive to increasing the gain of the game. In order to study the quantitative effect that the heterogeniety of the networks has on the gain, we use the method introduced in Section 2.2 by changing the rewiring times $K$. Figure V shows the effects that the rewiring times $K$ has on the population profit for game A which changes gradually from regular network to random network. We find that with the increment of the degree of the heterogeniety between game A and game B, the gain decreases gradually until to stabilization.

Figure VI demonstrates the effects that the adjustable parameter $\lambda$ of degree distributions has on the population gain. We see that the effect is not obvious, which shows that the heterogeneity of the networks has no obvious change when the network changes progressively from random network to a scale-free network.

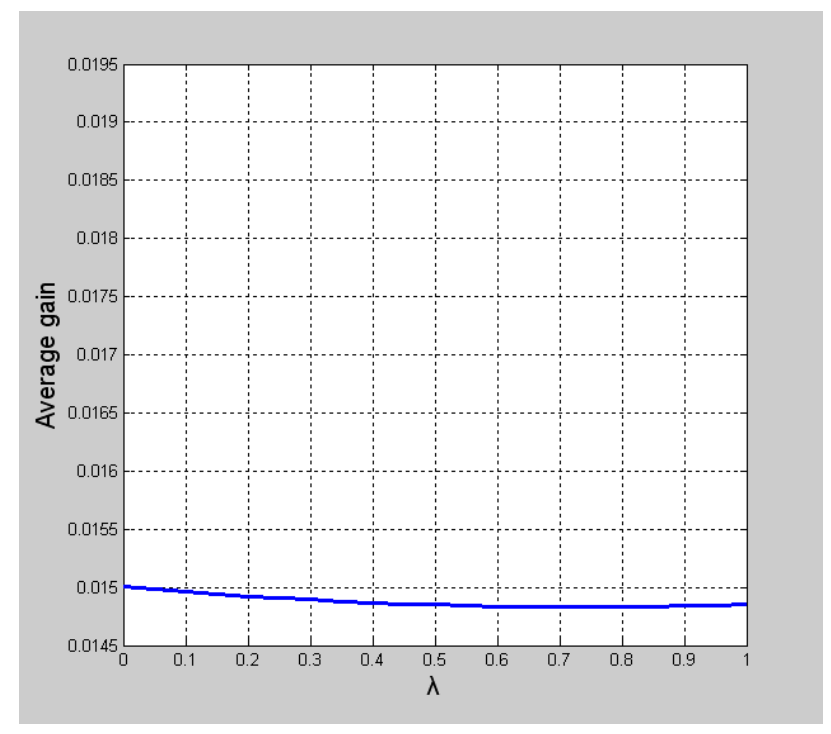

FIGURE VI. THE EFFECT THAT $\Lambda$ HAS ON THE AVERAGE GAIN OF THE POPULATION (THE NETWORK CARRIER CHANGES GRADUALLY FROM A SCALE-FREE NETWORK TO RANDOM NETWORK WITH THE INCREMENT OF $\Lambda$. M0 $=2, M=2$. OTHER PARAMETERS ARE THE SAME AS IN FIGURE 4)

Figure VII shows the parameter space in which paradox occurs on the two-dimensional lattice. The calculation results demonstrate that when game A uses a two-dimensional lattice (heterogeneous with game B), the parameter space where the strong paradox occurs is slightly larger than that in other three networks while the parameter space where the weak paradox occurs is slightly smaller than that in other three networks. Therefore, for the two-dimensional network, homogeneousness between game $\mathrm{A}$ and game $\mathrm{B}$ enlarges the parameter space in strong paradox. 


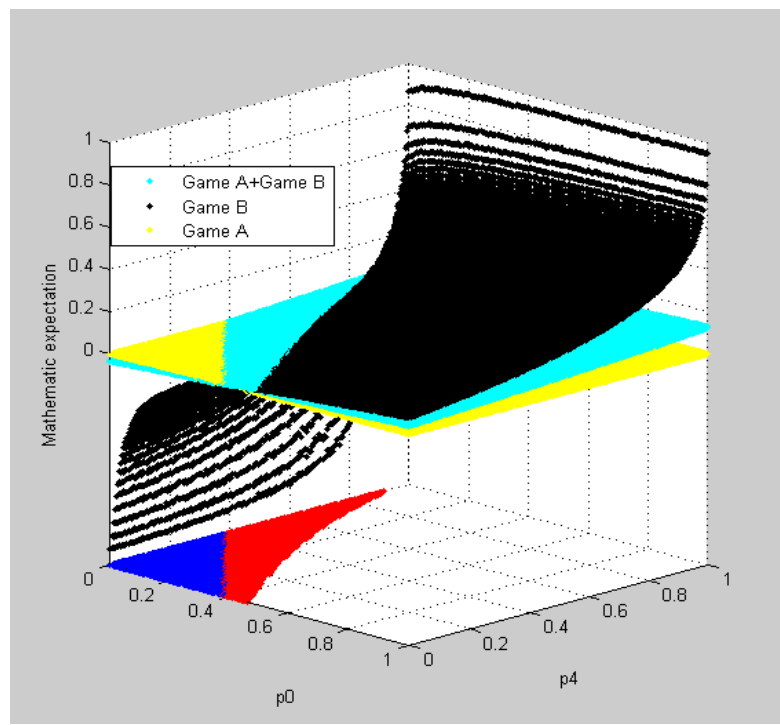

a. random network

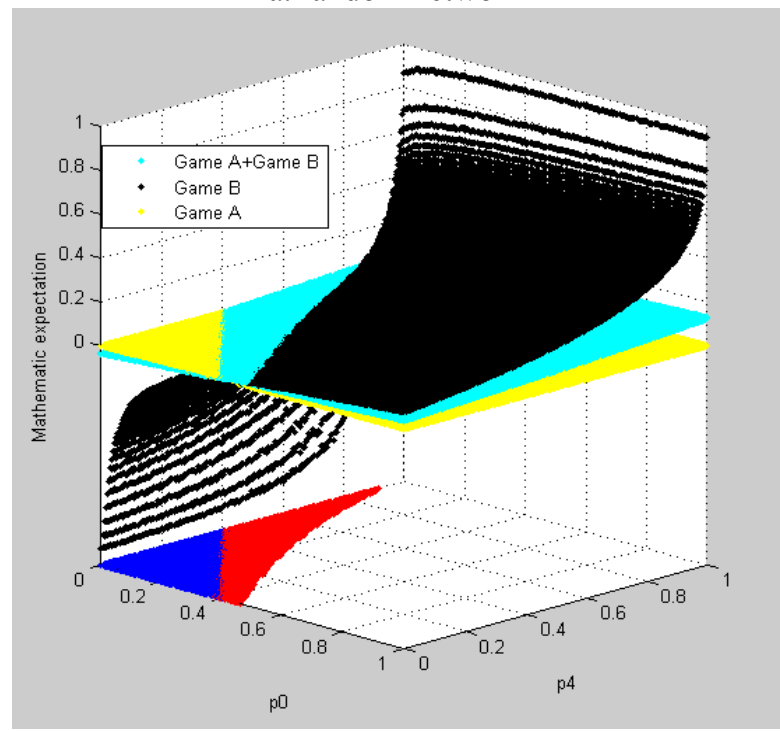

b. two-dimensional lattice

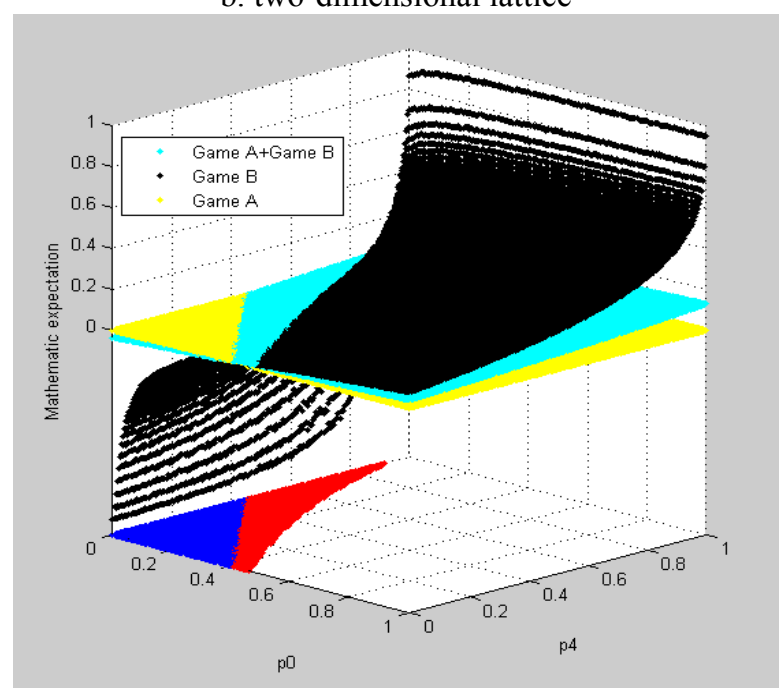

c. BA scale-free network

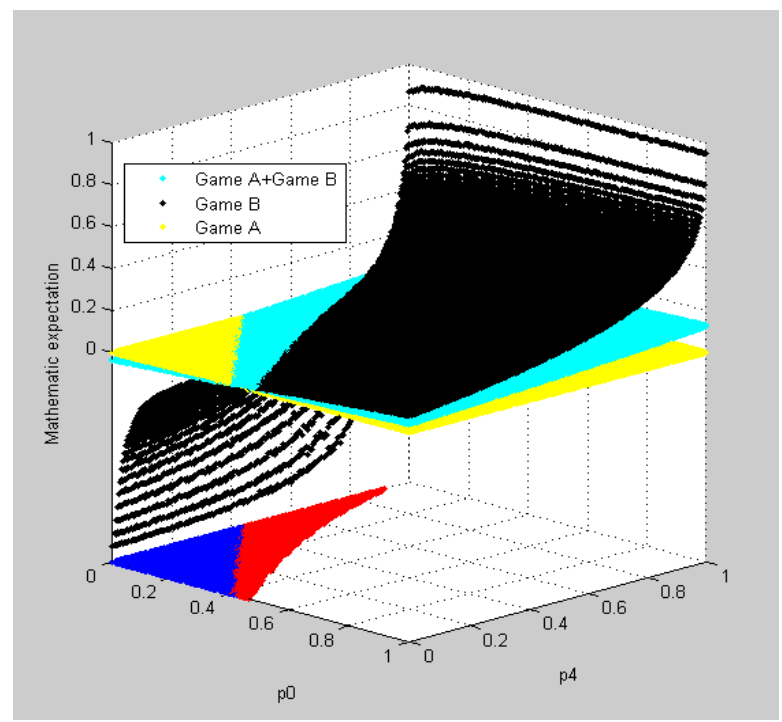

d. fully-connected network

FIGURE VII. THE RESULTS OF PARRONDO'S GAME BASED ON TWO-DIMENSIONAL NETWORKS (A. GAME A USES TWODIMENSIONAL LATTICE B. GAME A USES RANDOM

NETWORK. THE REWIRING TIMES ARE 1600. C. GAME A USES BA SCALE-FREE NETWORK. D. GAME A USES FULLYCONNECTED NETWORK. THE POPULATION SIZE IS N $=1600$. $\Gamma=0.5$. THE PARAMETERS OF GAME B ARE $P 1=0.15$ AND $\mathrm{P} 2=\mathrm{P} 3=0.7$. THE BLUE AREA DENOTES THE PARAMETERS AREA OF WEAK PARRONDO'S PARADOX WHILE THE RED STANDS FOR THE STRONG ONE).

\section{CONCLUSIONS}

For the cases that the spatial networks between game $\mathrm{A}$ and game $\mathrm{B}$ are different (heterogeneous networks), this paper demonstrates the effects of the heterogeneity of the networks through the theoretical analysis and computation simulations.

- We perform the theoretical analysis on a onedimensional line. Game A uses the following two networks: a one-dimensional line (homogeneous with game B) and a fully-connected network (heterogeneous with game $\mathrm{B}$ ). We deduce the transition probability matrix of game A under these two networks and give the mathematical expectation of the randomized game $\mathrm{A}+\mathrm{B}$. The theoretical results show that when game A uses a one-dimensional line (homogeneous with game $\mathrm{B}$, the parameter space where strong paradox occurs is smaller than that in fully-connected network (heterogeneous with game B). Therefore, for the one- dimensional line, the heterogeneity between game A and game B enlarges the parameter space where the strong paradox occurs.

- We perform computer simulations on twodimensional networks. Game A uses the following four networks: a two-dimensional lattice (homogeneous with game B), random network, a BA scale-free network and a fully-connected network (the latter three network are heterogeneous with game B). The simulation results show that when game A uses the two-dimensional lattice (homogeneous with game 
B), the gain and the parameter space where the strong paradox occurs are both slightly larger than those in the other three networks. Moreover, the gain and the strong paradox space decrease with the increment of the degree of heterogeneity, which shows that the homogeneousness between game $\mathrm{A}$ and game $\mathrm{B}$ is beneficial for two-dimensional networks.

\section{ACKNOWLEDGMENT}

This project was supported by The Ministry of Education, Humanities and Social Sciences research projects (Grant No. 13YJAZH106 and 15YJCZH210); The Talent Project for Higher Education Promotion Program of Anhui Province; and Anhui Provincial Foundation for Outstanding Young Teachers in Higher Education Institutions, China (Grant No. 2013SQRL023ZD);Anhui Provincial Natural Science Foundati on (Grant No. 1708085MF164).

\section{REFERENCES}

[1] J. M. R. Parrondo, "How to cheat a bad mathematician, in EEC HC\&M Network on Complexity and Chaos (\#ERBCHRX-CT940546), ” ISI, Torino, Italy, 1996,Unpublished.

[2] G. P. Harmer and D. Abbott, "Parrondo's paradox," Statistical Science, vol. 14, pp. 206-213, 1999.

[3] G. P. Harmer and D. Abbott, "Losing strategies can win by Parrondo's paradox," Nature, vol. 402, pp. 864,1999.

[4] D. Abbott, "Asymmetry and Disorder: A Decade of Parrondo's Paradox," Fluctuation and Noise Letters, vol. 9, pp. 129-156, 2010.

[5] J. M. R. Parrondo, G. P. Harmer and D. Abbott, "New paradoxical games based on Brownian ratchets," Physical Review Letters, vol. 85, pp. 5226-5229, 2000.

[6] R. J. Kay and N. F. Johnson, "Winning combinations of historydependent games," Physical Review E, vol. 67, pp. 056128, 2003.

[7] P. Arena, S. Fazzino, L. Fortuna and P. Maniscalco, "Game theory and non-linear dynamics: the Parrondo paradox case study," Chaos Solitons \& Fractals, vol. 17, pp. 545-555, 2003.

[8] R. Toral, "Cooperative Parrondo's games," Fluctuation and Noise Letters, vol. 1, pp. 7-12, 2001.

[9] Z. Mihailovic and M. Rajkovic, "One dimensional asynchronous cooperative Parrondo's games," Fluctuation and Noise Letters, vol. 3, pp. 389-398, 2003.

[10] Z. Mihailovic and M. Rajkovic, "Cooperative Parrondo's games on a two- dimensional lattice," Physica A, vol. 365, pp. 244-251,2006.

[11] C. Wang, N. G. Xie, L. Wang, Y. Ye and G. Xu, “A Parrondo's paradox game depending on capital parity," Fluctuation and Noise Letters, vol. 10,pp. 147-156, 2011.

[12] R. Toral, "Capital redistribution brings wealth by Parrondo's paradox," Fluctuation and Noise Letters, vol. 2, pp. 305-311, 2002.

[13] N.G. Xie, Y. Chen, Y. Ye, G. Xu, L.G. Wang and C. Wang, "Theoretical analysis and numerical simulation of Parrondo's paradox game in space," Chaos, Solitons \& Fractals, vol. 44, pp. 401-414, 2011.

[14] L. G. Wang, N. G. Xie, G. Xu, C. Wang, Y. Chen and Y. Ye, "Gamemodel research on coopetition behavior of Parrondo's paradox based on network," Fluctuation and Noise Letters, vol. 10, pp. 77-91, 2011.

[15] Y. Ye, N. G. Xie, L.G. Wang, L. Wang and Y. W. Cen, "Cooperation and competition in history-dependent Parrondo's game on networks," Fluctuation and Noise Letters, vol. 10, pp. 323-336, 2011

[16] Y. Ye, N. G. Xie, L. G. Wang, R. Meng and Y. W. Cen, "Study of biotic evolutionary mechanisms based on the multi-agent Parrondo's games," Fluctuation and Noise Letters, vol. 11, pp. 352-364, 2012.

[17] G. Szabo, A. Szolnoki and R.Izsak, "Rock-scissors-paper game on regular small-world networks, "Journal of Physics A:Mathematical and General, vol. 37, pp. 2599-2609, 2004.
[18] J. Gómez-Gardeñes and Y. Moreno, "From scale-free to Erdos-Rényi networks,” Physical Review E, vol. 73, pp. 645-666, 2006. 